\title{
Development of an insecticide impregnated polymer wall lining for malaria vector control
}

\author{
Mthokozisi Sibanda*, Walter Focke \\ From Challanges in malaria research: Core science and innovation \\ Oxford, UK. 22-24 September 2014
}

\begin{abstract}
Background
Dichlorodiphenyltrichloroethane (DDT), pyrethroids, organophosphates and carbamates are insecticides recommended by the World Health Organisation (WHO) for malaria vector control [1]. Long Life Insecticide-Treated Nets (LLIN) and Indoor Residual Spray (IRS) are the flagship interventions recommended by the WHO for malaria control. LLIN employ pyrethroids as active ingredients [2]. IRS is most effective when using DDT, a persistent organic pollutant [3]. Insecticide Treated Wall Lining (ITWL) or Netlon ${ }^{\circledR}$ is a slow release technology that combines the advantages of LLIN and IRS. Current commercial wall linings are produced using labour intensive fabric weaving methods. This study explored the effectiveness of inexpensive monofilament mesh linings produced by extruding insecticide impregnated polyethylene and poly(ethyleneco-vinyl acetate) (EVA) directly into a net format.
\end{abstract}

\section{Materials and methods}

Polyethylene and EVA were extruded with pyrethroids (deltamethrin and alphacypermethrin) and organophosphates (ca. 0.5 wt.\% insecticide) respectively through a dye to form monofilament meshes. Scanning Electron Microscopy (SEM) and Confocal Fluorescence Microscopy (CFM) was used to characterize the presence of insecticide on the surface of the monofilaments. Efficacy testing was done using standard WHO bioassay tube tests.

\section{Results}

For Netlon ${ }^{\circledR}$ to be effective, the insecticides have to be available on the monofilament surface since they are contact poisons. SEM and CFM micrographs show that pyrethroids are present and evenly distributed on the surface of the monofilament mesh. The WHO effectiveness criterion is a mortality exceeding $80 \%$ after 24 hours following a
30 minute exposure of $A n$. arabiensis mosquitoes to test samples. The pyrethroid impregnated mesh samples achieved 100\% mortality in this test even after 24 months of storage. Samples impregnated with organophosphates failed to achieve the WHO criterion. Poor performance of the organophosphate insecticides in the EVA matrix was probably due to the high solubility of these insecticides in this polymer.

\section{Conclusions}

Monofilament polyethylene mesh impregnated with pyrethroid insecticides was successfully produced by a simple direct extrusion technique. It is an inexpensive method for the production of slow-release polymer-based wall linings. Laboratory efficacy tests suggest that this ITWL technology may be a potential substitute for IRS and it may also complement LLIN.

Published: 22 September 2014

\section{References}

1. World Health Organisation: WHO insecticides approved for indoor residual spraying against malaria vectors. Geneva: World Health Organisation; 2013.

2. World Health Organisation: WHO recommended long-lasting insecticidal nets. Geneva: World Health Organisation; 2014.

3. Sibanda MM, Focke WW: Degradation of insecticides used for indoor spraying in malaria control and possible solutions. Malar J 2011, 10:307.

doi:10.1186/1475-2875-13-S1-P80

Cite this article as: Sibanda and Focke: Development of an insecticide impregnated polymer wall lining for malaria vector control. Malaria Journal 2014 13(Suppl 1):P80. 